ARTICLE

\title{
On-the-Fly Monte Carlo Dominance Ratio Calculation Using the Noise Propagation Matrix
}

\author{
Thomas M. SUTTON ${ }^{1, *}$, Paul K. ROMANO ${ }^{2}$ and Brian R. NEASE ${ }^{3}$ \\ ${ }^{1}$ Knolls Atomic Power Laboratory - Bechtel Marine Propulsion Corporation, Schenectady, NY, USA \\ ${ }^{2}$ Massachusetts Institute of Technology, Cambridge, MA, USA \\ ${ }^{3}$ Bettis Atomic Power Laboratory - Bechtel Marine Propulsion Corporation, Pittsburgh, PA, USA
}

\begin{abstract}
Two Monte-Carlo-based methods for computing the dominance ratio in reactor calculations are the Fission Matrix Method (FMM) and the Coarse Mesh Projection Method (CMPM). The FMM allows an estimate of the dominance ratio to be computed before the fission source has converged, but requires a fine mesh - and hence considerable computational resources - for sufficient accuracy. Conversely, the CMPM gives very accurate results on a coarse mesh with very little computational effort, but can be used only after the fission source has converged. In this paper we describe a new method called the Noise Propagation Matrix Method (NPMM) that has the same coarse-mesh accuracy properties as the CMPM while also permitting an 'on-the-fly' estimation of the dominance ratio during fission source convergence. Like the CMPM, the NPMM uses the noise propagation matrix (NPM) in determining the dominance ratio. The two methods differ, however, in how the matrix is used to obtain the dominance ratio. A new derivation of the equations used to compute the NPM in a Monte Carlo calculation is presented that eliminates an approximation made in an earlier work on the CMPM. It is shown that by using the improved expression for the NPM, the dominance ratio can be found directly and simply as the largest-modulus eigenvalue of the matrix - thereby eliminating the more complicated time-series-analysis method used by the CMPM. Results for several problems are presented that demonstrate the validity of the method.
\end{abstract}

KEYWORDS: Monte Carlo, dominance ratio

\section{Introduction}

The dominance ratio of a fissioning system is defined as the first harmonic eigenvalue divided by the fundamental mode eigenvalue. Its significance lies in two distinct areas. First, it is a property of the physical system, and hence is related to such aspects of the system as xenon stability. Second, it is a property of the transport equation, and hence is related to such aspects of the solution method as the fission source convergence rate and cycle-to-cycle correlation. Being able to determine the dominance ratio is thus important to reactor designers and analysts as well as reactor physics methods developers. Accurate determination of the dominance ratio from Monte Carlo calculations has been the subject of considerable study in recent years. ${ }^{1-5)}$

For many applications it is only necessary that the dominance ratio be an end result of the Monte Carlo calculation. There are some applications, however, in which an evolving estimate of the dominance ratio obtained 'on-the-fly' during the calculation may be useful. It is known, for example, that using the standard power method for fission source iterations results in the tallies from successive fission generations (cycles) being correlated, and that this correlation must either be minimized or accounted for in order to determine accurate statistical uncertainties. ${ }^{6)}$ It is also known that the degree of correlation can be estimated

*Corresponding author, E-mail:Thomas.Sutton@unnpp.gov from the dominance ratio. One method of minimizing the correlation between successive tallies is by combining multiple successive fission cycles into batches, and defining the tally variables over the batches rather than over the cycles. ${ }^{7)}$ Here, an estimate of the dominance ratio obtained during the inactive cycle phase of the calculation is helpful in automatically determining the number of cycles per batch to use during the active cycle phase.

One means of estimating the dominance ratio using Monte Carlo is the fission matrix method (FMM) ${ }^{8)}$ The FMM involves the division of the configuration space of the problem into a number of discrete cells. Element $(i, j)$ of the fission matrix is defined as the expected number of direct progeny fission neutrons born in cell $i$ due to a parent fission neutron born in cell $j$. An estimate of the fission matrix may be easily obtained in Monte Carlo criticality calculations, and its eigenvalue spectrum determined by standard matrix algebra methods. The dominance ratio may then be estimated as the ratio of the first-harmonic eigenvalue of the matrix to that of the fundamental mode. While the fundamental-mode eigenvalue of the fission matrix is a good estimate of the fundamental-mode eigenvalue of the Monte Carlo calculation, the same is not generally true of the first-harmonic eigenvalue and thus the dominance ratio will be in error. ${ }^{5)}$ The error in the first-harmonic eigenvalue of the fission matrix is due to the spatial discretization, and may be reduced by refinement of the mesh. However, the corresponding increases in computer memory requirements 
and computational effort severely limit the usability of the FMM for practical applications. It should be noted, however, that the FMM has the desirable characteristic that a rough estimate of the dominance ratio may be computed prior to fission source convergence. ${ }^{5)}$ This gives a Monte Carlo code with a FMM capability the ability to estimate the dominance ratio during the source convergence process.

The Coarse Mesh Projection Method (CMPM) — which evolved from a method based on the use of an autoregressive-moving average $(2,1)(\operatorname{ARMA}(2,1))$ fit-was developed to overcome the accuracy limitations of the FMM when used with a small number of large mesh cells. ${ }^{1-4)}$ Like the FMM, the CMPM uses a spatial discretization. During the Monte Carlo calculation, quantities are tallied that will later be used to compute an estimate of the noise propagation matrix (NPM). At the completion of the Monte Carlo calculation, the NPM is determined and its left (adjoint) eigenvector corresponding to the eigenvalue with the largest modulus is calculated. The time series formed by taking the inner product of this eigenvector and the source vector at each generation is an autoregressive order 1 process with a correlation coefficient equal to the dominance ratio. The CMPM estimates the dominance ratio by computing this correlation coefficient. Unlike the case of the FMM, however, the CMPM generally produces an accurate estimate of the dominance ratio even for very coarse mesh. The only disadvantage of the CMPM (at least in its reported implementation to date) relative to the FMM is that the dominance ratio may not be computed until the fission source has fully converged. ${ }^{5)}$

This paper introduces a new algorithm - termed the Noise Propagation Matrix Method (NPMM) - for computing the dominance ratio in Monte Carlo analyses. Like the CMPM, the method uses a determination of the NPM by the Monte Carlo code. The essence of the NPMM is that once an estimate of the NPM is obtained, the dominance ratio is very simply found as the largest-modulus eigenvalue of the NPM.

There are three aspects to the work reported on in this paper. First, it is shown that the mathematical derivation previously used to obtain the expressions governing the practical implementation of the CMPM can be improved upon, thus yielding an improved estimate of the NPM. Second, it is shown that the largest-modulus eigenvalue of this NPM provides an estimate of the dominance ratio that is identical to that which would result from the time series analysis of the CMPM had the same NPM been used. Finally, it is demonstrated that this method allows a reasonable estimate of the dominance ratio to be determined prior to the convergence of the fission source. This method thus retains the coarse-mesh accuracy and computational efficiency of the CMPM while also permitting an 'on-the-fly' determination of the dominance ratio during source convergence.

\section{The Noise Propagation Matrix}

As mentioned in Section I, both the CMPM and the NPMM use a Monte-Carlo-generated NPM in computing the dominance ratio. The NPM is not tallied directly, but instead is computed from two source correlation matrices that are tallied during the Monte Carlo calculation. In this section, two different expressions are derived that relate the NPM to the correlation matrices. The first such expression was originally derived by Nease, and has been used to implement the CMPM in the MCNP Monte Carlo code. ${ }^{4,5)}$ The second expression-derived for the first time in this paper-has been used in the implementation of the NPMM in the MC21 code. ${ }^{9)}$ It is shown that the new derivation avoids an approximation used in the original derivation.

Implementations of the CMPM and the NPMM to-date utilize a rectangular mesh superimposed on the Monte Carlo geometry. A discretized representation of the fission source is obtained by tallying the neutron production rate due to fission in each of the mesh cells. Let $\mathbf{S}^{(m)}$ denote the fission source vector at the conclusion of fission-iteration cycle $m$, where an element of the vector corresponds to the fission source in one of the cells. It is assumed that $m$ is sufficiently large that the source vector can be considered a stationary random variable. Following Nease and Ueki, we decompose the fission source vector as

$$
\mathbf{S}^{(m)}=N \mathbf{S}_{0}+\sqrt{N} \mathbf{e}^{(m)},
$$

where $N$ is the number of neutrons per cycle, $\mathbf{S}_{0}$ is the fundamental-mode fission source vector normalized to the fundamental-mode eigenvalue, and $\mathbf{e}^{(m)}$ is a stochastic noise vector that represents the deviation of the cycle- $m$ fission source vector from its expected value. ${ }^{3)}$ Denoting the ensemble-averaging process by $\langle\ldots\rangle$, Eq. (1) leads to

$$
\left\langle\mathbf{S}^{(m)}\right\rangle=N \mathbf{S}_{0} \equiv \mathbf{S}
$$

Taking the ensemble average of Eq. (1) and using Eq. (2) yields

$$
\left\langle\mathbf{e}^{(m)}\right\rangle=\mathbf{0}
$$

Nease and Ueki show that to lowest order in the noise terms, $\mathbf{e}^{(m)}$ is propagated from cycle to cycle according to

$$
\mathbf{e}^{(m+1)}=\mathbf{A}_{0} \mathbf{e}^{(m)}+\boldsymbol{\varepsilon}^{(m+1)},
$$

where $\mathbf{A}_{0}$ is the NPM and $\boldsymbol{\varepsilon}^{(m+1)}$ is a vector representing the noise introduced at cycle $m+1$. This latter quantity has the property

$$
\left\langle\boldsymbol{\varepsilon}^{(m+1)}\right\rangle=\mathbf{0}
$$

In Refs. 3) and 4), covariance matrices are defined in terms of the noise vector $\mathbf{e}^{(m)}$. Expressions are then found relating these covariance matrices to the NPM. To-date, however, practical implementations of the CMPM and NPMM in production Monte Carlo codes have used an alternative methodology which utilizes correlation matrices defined in terms of the fission source vector $\mathbf{S}^{(m)} .^{5)}$ In this paper we only consider this latter methodology.

Multiplying Eq. (4) through by $\sqrt{N}$, adding $N \mathbf{S}_{0}$ to 
both sides, and using the property $\mathbf{A}_{0} \mathbf{S}_{0}=\mathbf{0}$, it can be shown that the equation that governs the propagation of the fission source vector from cycle to cycle is ${ }^{4)}$

$$
\mathbf{S}^{(m+1)}=\mathbf{A}_{0} \mathbf{S}^{(m)}+\boldsymbol{\eta}^{(m+1)},
$$

where

$$
\boldsymbol{\eta}^{(m)} \equiv N \mathbf{S}_{0}+\sqrt{N} \boldsymbol{\varepsilon}^{(m)} .
$$

Multiplying Eq. (6) through from the right by $\mathbf{S}^{(m) \mathrm{T}}$ (where ' $\mathrm{T}$ ' denotes transpose) and ensemble-averaging results in

$$
\left\langle\mathbf{S}^{(m+1)} \mathbf{S}^{(m) \mathrm{T}}\right\rangle=\mathbf{A}_{0}\left\langle\mathbf{S}^{(m)} \mathbf{S}^{(m) \mathrm{T}}\right\rangle+\left\langle\boldsymbol{\eta}^{(m+1)} \mathbf{S}^{(m) \mathrm{T}}\right\rangle .
$$

The source correlation matrices are defined as

$$
\mathbf{L}_{0}^{\prime} \equiv\left\langle\mathbf{S}^{(m)} \mathbf{S}^{(m) \mathrm{T}}\right\rangle
$$

and

$$
\mathbf{L}_{1}^{\prime} \equiv\left\langle\mathbf{S}^{(m+1)} \mathbf{S}^{(m) \mathrm{T}}\right\rangle
$$

With these definitions, Eq. (8) can be rearranged to obtain an expression relating the correlation matrices to the NPM

$$
\mathbf{A}_{0}=\left[\mathbf{L}_{1}^{\prime}-\left\langle\boldsymbol{\eta}^{(m+1)} \mathbf{S}^{(m) \mathrm{T}}\right\rangle\right]\left(\mathbf{L}_{0}^{\prime}\right)^{-1} .
$$

Up to this point, the derivation of the expression for the NPM in terms of the source vector is the same as given in Ref. 4). In that work, the derivation was continued from this point by making the approximation

$$
\left\langle\boldsymbol{\eta}^{(m+1)} \mathbf{S}^{(m) \mathrm{T}}\right\rangle \approx \mathbf{L}_{0}^{\prime} .
$$

Substitution of Eq. (12) into Eq. (11) then produced

$$
\hat{\mathbf{A}}_{0}=\mathbf{L}_{1}^{\prime}\left(\mathbf{L}_{0}^{\prime}\right)^{-1}-\mathbf{I},
$$

where we have introduced the symbol $\hat{\mathbf{A}}_{0}$ to represent this approximation to the NPM. This expression for the NPM is the one used for the CMPM implementation in the MCNP code. $^{4,5)}$

We now show that the approximation given by Eq. (12) can be avoided. Using Eqs. (1) and (7), we have

$$
\left\langle\boldsymbol{\eta}^{(m+1)} \mathbf{S}^{(m) \mathrm{T}}\right\rangle=\left\langle\left(N \mathbf{S}_{0}+\sqrt{N} \boldsymbol{\varepsilon}^{(m+1)}\right)\left(N \mathbf{S}_{0}+\sqrt{N} \mathbf{e}^{(m)}\right)^{\mathrm{T}}\right\rangle .
$$

As shown in Refs. 3) and 4), the noise introduced in one cycle is uncorrelated with the accumulated noise term from all previous cycles, i.e.

$$
\left\langle\boldsymbol{\varepsilon}^{(m+1)} \mathbf{e}^{(m) \mathrm{T}}\right\rangle=0 .
$$

Using Eqs. (2), (3), (5) and (15), Eq. (14) may be simplified to

$$
\left\langle\boldsymbol{\eta}^{(m+1)} \mathbf{S}^{(m) \mathrm{T}}\right\rangle=\mathbf{S S}^{\mathrm{T}} .
$$

Note that unlike Eq. (12), this expression does not involve any additional approximation beyond those already employed in arriving at Eq. (4). Substituting Eq. (16) into Eq. (11) yields the improved expression for the NPM

$$
\mathbf{A}_{0}=\left[\mathbf{L}_{1}^{\prime}-\mathbf{S S}^{\mathrm{T}}\right]\left(\mathbf{L}_{0}^{\prime}\right)^{-1} .
$$

This expression for the NPM is the one used for the NPMM implementation in the MC21 code. Details of that implementation will be given in Section IV.

\section{Equivalence of the Dominance Ratio as Deter- mined by the CMPM and the NPMM}

In this section, it is shown that given correct and consistent formulations of the NPM, the dominance ratio as determined by the CMPM and NPMM algorithms are identical. As will be shown in the Appendix, however, the approximation used to obtain the formulation of the NPM given by Eq. (13) introduces extraneous terms that alter the eigenvalue spectrum to such an extent that is it not directly usable with the NPMM algorithm. The improved formulation given by Eq. (17), however, works equally well with either algorithm.

It has been shown that the dominance ratio given by the time-series analysis of the CMPM is equivalent to the ratio of two Frobenius inner products, i.e. ${ }^{4)}$

$$
\frac{k_{1}}{k_{0}}=\frac{\left(\mathbf{d}_{1} \mathbf{d}_{1}^{\mathrm{T}}\right): \mathbf{L}_{1}^{\prime}}{\left(\mathbf{d}_{1} \mathbf{d}_{1}^{\mathrm{T}}\right): \mathbf{L}_{0}^{\prime}}=\frac{\mathbf{d}_{1}^{\mathrm{T}} \mathbf{L}_{1}^{\prime} \mathbf{d}_{1}}{\mathbf{d}_{1}^{\mathrm{T}} \mathbf{L}_{0}^{\prime} \mathbf{d}_{1}},
$$

where $\mathbf{d}_{1}$ is the left eigenvector of $\mathbf{A}_{0}$ corresponding to the largest-modulus eigenvalue ${ }^{a} \lambda_{1}$, i.e.

$$
\mathbf{A}_{0}^{\mathrm{T}} \mathbf{d}_{1}=\lambda_{1} \mathbf{d}_{1} .
$$

Substituting Eq. (17) into Eq. (19), after some manipulation we obtain

$$
\mathbf{d}_{1}^{\mathrm{T}} \mathbf{L}_{1}^{\prime}-\mathbf{d}_{1}^{\mathrm{T}} \mathbf{S} \mathbf{S}^{\mathrm{T}}=\lambda_{1} \mathbf{d}_{1}^{\mathrm{T}} \mathbf{L}_{0}^{\prime} .
$$

To deal with the second term on the left side of the above equation, we use Eqs. (2) and (19) to get

$$
\mathbf{d}_{1}^{\mathrm{T}} \mathbf{S}=\frac{1}{\lambda_{1}}\left(\mathbf{A}_{0}^{\mathrm{T}} \mathbf{d}_{1}\right)^{\mathrm{T}} N \mathbf{S}_{0}=\frac{N}{\lambda_{1}} \mathbf{d}_{1}^{\mathrm{T}} \mathbf{A}_{0} \mathbf{S}_{0} .
$$

As discussed in Ref. 3) $\mathbf{A}_{0} \mathbf{S}_{0}=\mathbf{0}$, hence from Eq. (21) we obtain

$$
\mathbf{d}_{1}^{\mathrm{T}} \mathbf{S}=\mathbf{0} .
$$

Multiplying both sides of Eq. (20) from the right by $\mathbf{d}_{1}$ and using Eq. (22) we obtain

$$
\mathbf{d}_{1}^{\mathrm{T}} \mathbf{L}_{1}^{\prime} \mathbf{d}_{1}=\lambda_{1} \mathbf{d}_{1}^{\mathrm{T}} \mathbf{L}_{0}^{\prime} \mathbf{d}_{1},
$$

and hence

\footnotetext{
a To maintain consistency with earlier work, we use a subscript " 1 " to denote the largest-modulus eigenvalue of the NPM, $\lambda_{1}$, but use a subscript " 0 " to denote the largest-modulus eigenvalue of the transport equation, $k_{0}$.
} 


$$
\lambda_{1}=\frac{\mathbf{d}_{1}^{\mathrm{T}} \mathbf{L}_{1}^{\prime} \mathbf{d}_{1}}{\mathbf{d}_{1}^{\mathrm{T}} \mathbf{L}_{0}^{\prime} \mathbf{d}_{1}}
$$

Comparing Eq. (24) with Eq. (18), we see that

$$
\lambda_{1}=\frac{k_{1}}{k_{0}}
$$

i.e. the largest-modulus eigenvalue of the Monte Carlo estimate of the NPM is identically the dominance ratio that one obtains from the CMPM using that same NPM. The importance of this result is that one may compute the dominance ratio simply by using any standard method of finding the largest-modulus eigenvalue of $\mathbf{A}_{0}$, and that the value so obtained has the same favorable mesh-size properties as that obtained using the CMPM.

\section{Implementation of the NPMM in MC21}

This section provides a few details of how the NPMM method for calculating the dominance ratio has been implemented in the MC21 Monte Carlo code. ${ }^{9)}$ Let $m_{0}$ denote the number of cycles run before the tallying of the elements of the correlation matrix is begun. After cycle $m$ $\left(m>m_{0}+1\right)$, the following quantities have been accumulated:

$$
\begin{aligned}
\hat{\mathbf{L}}_{0}^{(m)} & \equiv \sum_{i=m_{0}+1}^{m} \mathbf{S}^{(i)} \mathbf{S}^{(i) \mathrm{T}}, \\
\hat{\mathbf{L}}_{1}^{(m)} & \equiv \sum_{i=m_{0}+2}^{m} \mathbf{S}^{(i)} \mathbf{S}^{(i-1) \mathrm{T}},
\end{aligned}
$$

and

$$
\hat{\mathbf{S}}^{(m)} \equiv \sum_{i=m_{0}+1}^{m} \mathbf{S}^{(i)}
$$

The cycle- $m$ estimate of the NPM is then computed as

$$
\mathbf{A}_{0}^{(m)}=\left(\frac{m-m_{0}}{m-m_{0}-1} \hat{\mathbf{L}}_{1}^{(m)}-\frac{1}{m-m_{0}} \hat{\mathbf{S}}^{(m)} \hat{\mathbf{S}}^{(m) \mathrm{T}}\right)\left(\hat{\mathbf{L}}_{0}^{(m)}\right)^{-1} .
$$

The largest-modulus eigenvalue of $\mathbf{A}_{0}^{(m)}$, and hence the cycle- $m$ estimate of the dominance ratio, is found using the SGEEV subroutine from LAPACK. ${ }^{10)}$

The default procedure in MC21 is to make $m_{0}$ the same as the number of discard cycles used to converge the fission source. In this case, $\mathbf{A}_{0}^{(m)}$ is computed-and the dominance ratio estimated-starting with the second non-discarded cycle. As will be seen in the next section, however, the NPMM seems to provide a reasonable estimate of the dominance ratio well before the fission source has converged. Thus, like the FMM but unlike the CMPM, the NPMM can provide on-the-fly estimation of the dominance ratio during the source convergence process.

\section{Results}

\section{Slab Problem}

This problem is a one-dimensional (1-D), 200-cm-thick
Table 1 Macroscopic Cross sections for the slab problem

\begin{tabular}{cc}
\hline Cross section & Value $\left(\mathrm{cm}^{-1}\right)$ \\
\hline$\Sigma_{\mathrm{t}}$ & 1.00 \\
$\Sigma_{\mathrm{s}}$ & 0.70 \\
$\Sigma_{\mathrm{a}}$ & 0.30 \\
$\Sigma_{\mathrm{f}}$ & 0.15 \\
$v \Sigma_{\mathrm{f}}$ & 0.39 \\
\hline
\end{tabular}

Table 2 Dominance ratio using ten mesh intervals for the slab problem

\begin{tabular}{ccc}
\hline Reference & NPMM & CMPM $^{4)}$ \\
\hline 0.9992 & $0.9990(06)$ & $0.9986(11)$ \\
\hline
\end{tabular}

slab with vacuum boundary conditions. It assumes isotropic scattering and one-group cross sections (provided in Table 1). The analytic diffusion theory solution for the $n$ th-harmonic eigenvalue is

$$
k_{n}=\frac{v \Sigma_{\mathrm{f}}}{\Sigma_{\mathrm{a}}+D B_{n}^{2}} ; \quad n=0,1, \ldots,
$$

where

$$
B_{n}=\frac{(n+1) \pi}{\tilde{L}},
$$

is the geometric buckling,

$$
\tilde{L}=L+2 \cdot 0.7104 \cdot \lambda_{\text {tr }}
$$

is the slab thickness $L$ plus twice the distance to the extrapolated boundary, and $\lambda_{\text {tr }}$ is the transport mean free path. For the case of isotropic scattering considered here, $\lambda_{t r}=\Sigma_{t}^{-1}$ and $D=\left(3 \Sigma_{\mathrm{t}}\right)^{-1}$. Using Eq. (30), one obtains $k_{0}=1.299649$ and $k_{1}=1.298596$, giving a reference dominance ratio of 0.999190 . An additional reference solution was generated using the PARTISN discrete ordinates code with 5,000 spatial mesh cells and an $S_{200}$ quadrature to compute $k_{0}$, and then estimating the dominance ratio as $\left(k_{0} / k_{\infty}\right)^{3}$, where $k_{\infty}$ is the infinite-medium multiplication factor. ${ }^{11,12)}$ This procedure also yields a dominance ratio of 0.999190.

This problem was analyzed using MC21 with 20,000 neutrons per cycle, 8,000 discard cycles and 20,000 active cycles. The NPM was calculated during the 20,000 active cycles. Table 2 gives the reference dominance ratio, as well as the values obtained using the NPMM and the CMPM. ${ }^{4}$ The NPMM and CMPM calculations were run using ten equally-spaced mesh intervals across the slab. The values in parentheses following the dominance ratio results are two-standard-deviation uncertainties in the least-significant digits, where the variance is determined using 
Table 3 Effect of the number of mesh intervals on the dominance ratio for the slab problem

\begin{tabular}{ccc}
\hline $\begin{array}{c}\text { Number of } \\
\text { mesh } \\
\text { intervals }\end{array}$ & $\begin{array}{c}\text { NPMM } \\
\text { dominance } \\
\text { ratio }\end{array}$ & $\begin{array}{c}\text { Difference } \\
\text { from } \\
\text { reference } \\
\text { solutions }\end{array}$ \\
\hline 2 & $0.9986(7)$ & -0.0006 \\
3 & $0.9989(6)$ & -0.0003 \\
4 & $0.9989(6)$ & -0.0003 \\
5 & $0.9989(6)$ & -0.0003 \\
10 & $0.9990(6)$ & -0.0002 \\
20 & $0.9990(6)$ & -0.0002 \\
\hline
\end{tabular}

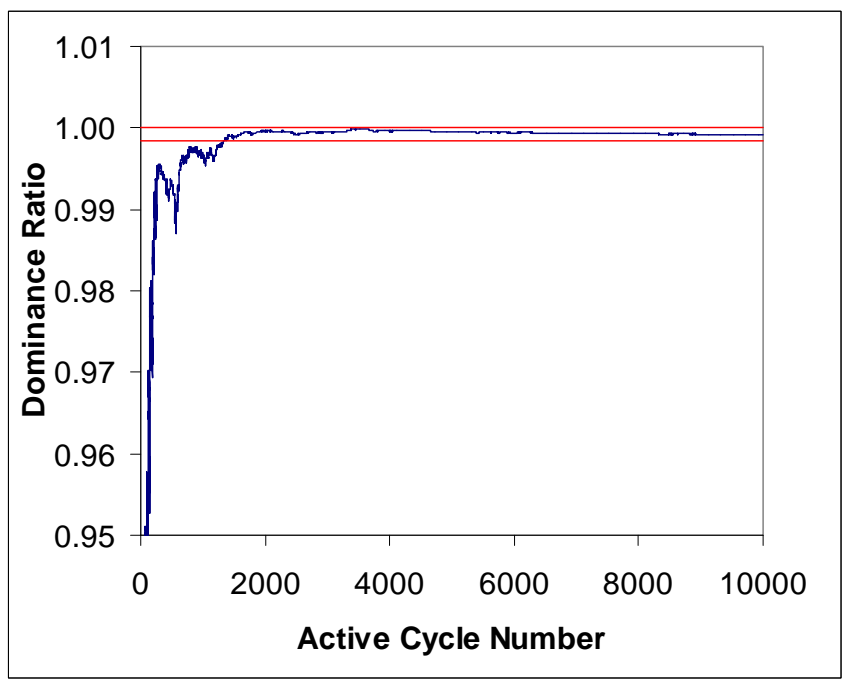

Fig. 1 Convergence of the dominance ratio for the slab problem

$$
\sigma^{2}=\frac{1}{M}\left(1-\frac{k_{1}^{2}}{k_{0}^{2}}\right),
$$

with $M=m-m_{0}$ being the number of cycles over which the NPM is computed. This formula was derived for the CMPM based on a result from time series analysis due to Box and Jenkins. ${ }^{4}$ (13) Since-as demonstrated in Section III- the NPMM result is identical to the result that one would have obtained from the CMPM with the same NPM, it follows that the variance of the NPM result should be the same as that of the CMPM result and hence the use of Eq. (33) is appropriate for both methods. The NPMM result agrees well within the statistical uncertainty with both the reference value and the CMPM result. Table 3 shows the results for six MC21 calculations where the number of mesh intervals was varied. Even with just two mesh cells, the NPMM result is in agreement with the reference value.

Figure 1 is a plot of the dominance ratio versus active cycle number, along with the $\pm 2 \sigma$ band about the final value. Convergence is initially very rapid, reaching two-significant-figure accuracy within about 200 cycles. To attain three-significant-figure accuracy, however, requires over 1,300 cycles.

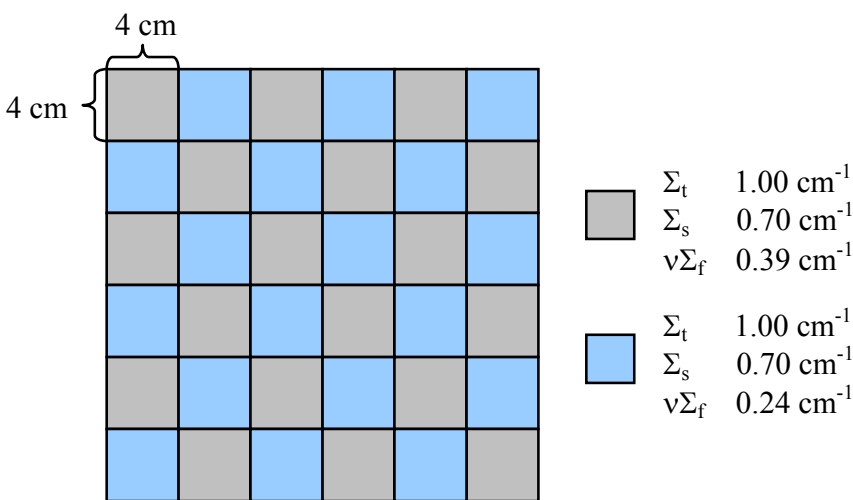

Fig. 2 2-D checkerboard problem geometry and nuclear data

Table 4 Dominance ratio results for the 2-D checkerboard problem

\begin{tabular}{ccll}
\hline Method & Mesh & Dominance ratio & $\begin{array}{c}\text { Difference } \\
\text { from } \\
\text { discrete } \\
\text { ordinates }\end{array}$ \\
\hline Discrete ordinates & & 0.9581 & \\
FMM & $48 \times 48$ & 0.9574 & -0.0007 \\
CMPM (Nease) & $6 \times 6$ & $0.9570(029)$ & -0.0011 \\
CMPM (MCNP) & $6 \times 6$ & $0.9481(101)$ & -0.0100 \\
NPMM & $6 \times 6$ & $0.9596(028)$ & +0.0015 \\
\hline
\end{tabular}

\section{Two-Dimensional Checkerboard Problem}

This problem consists of a two-dimensional (2-D), $6 \times 6$ array of two types of square regions arranged in a checkerboard pattern. Vacuum boundary conditions apply to all four sides. The problem employs one-group cross sections and isotropic scattering. The geometry and cross section data are given in Fig. 2. This problem was analyzed with MC21 using 400 discard cycles and 40,000 active cycles of 80,000 neutrons each, and a $6 \times 6$ mesh. Table 4 compares the dominance ratio obtained using the NPMM in MC21 with that obtained using the CMPM, a spectral radius analysis of a discrete ordinates calculation, and a FMM calculation using a highly-resolved $48 \times 48$ mesh. Two CMPM results are given. The first is from a Monte Carlo code used by Nease for his PhD thesis. ${ }^{4)}$ The NPM used for that calculation is equivalent to that used by the NPMM in MC21 and given by Eq. (17). The other CMPM result is from an MCNP calculation, and was obtained using the NPM given by Eq. (13). As can be seen, the NPMM result agrees with the reference discrete ordinates and fine-mesh FMM results at the two-standard-deviation level. Both CMPM results also agree with the reference calculations at the two-standard-deviation level. Note that although we compare to the discrete ordinates solution, its degree of accuracy is unknown.

\section{Large Two-Dimensional Problem}

This problem is a $2-\mathrm{D}, 18 \times 18$ array of three types of square regions with vacuum boundary conditions. ${ }^{6)}$ The geometry and cross section data are given in Fig. 3. This problem was analyzed using MC21 with 10,000 discard and 

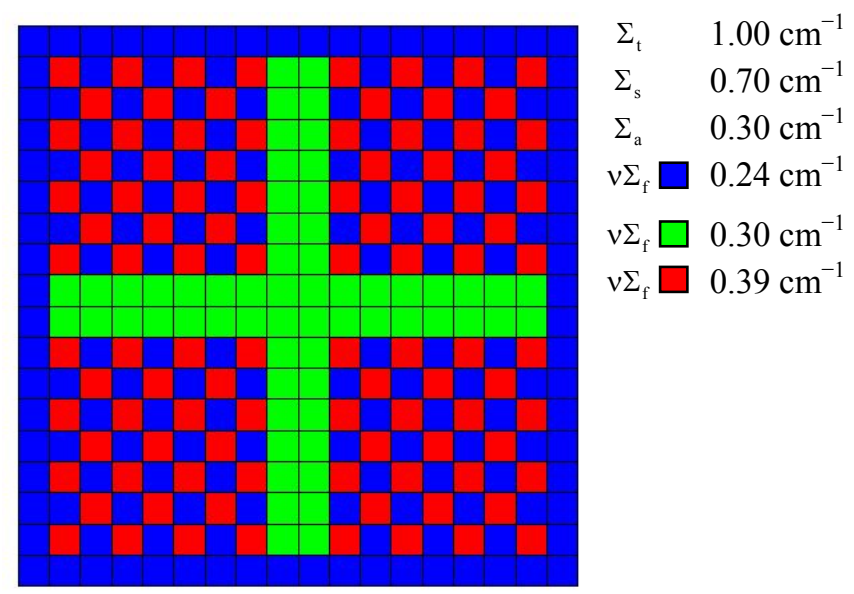

Fig. 3 Large 2-D problem geometry and nuclear data

40,000 active cycles of 100,000 neutrons each, and a $4 \times 4$ discretization. The dominance ratio results are given in Table 5, along with results obtained using the FMM, the CMPM as implemented in MCNP, two PARTISN calculations using a $360 \times 360$ mesh and $S_{16}$ quadrature, and the $\operatorname{ARMA}(2,1)$ method. ${ }^{6}$ ) Both PARTISN calculations estimated the first harmonic eigenvalue using core symmetry, with one using a vacuum boundary condition and the other an extrapolated zero-flux boundary condition on the symmetry plane. The FMM results show the strong dependence of the accuracy of this method on mesh size. It is possible that an even finer mesh than the finest shown here would change the result in the third significant digit. Therefore, the FMM results do not constitute a reference calculation, but are merely suggestive. The CMPM result is only given to three significant digits, but is in statistical agreement with the NPMM result. Both the ARMA $(2,1)$ and the NPMM results are given to four significant digits. Even though they do not quite agree to within the combined two-standard-deviation uncertainty, they do agree to three significant digits. The NPMM result is in good agreement with both PARTISN results.

Figure 4 shows two plots of the dominance ratio estimate for this problem as a function of cycle number. In one case, the default procedure was used in which computation of the NPM and dominance ratio is not begun until the discard cycles have been completed and the source is fully converged. Note that in this calculation 10,000 initial cycles were discarded to minimize the possibility of non-convergence of the source for the purpose of this comparison. In typical calculations, such a large number of discard cycles is not necessary. In the other case, the computation of the NPM and dominance ratio is begun immediately with the second discard cycle. The trajectories of the dominance ratio versus cycle number are very similar in the two cases, indicating that the NPMM can yield an accurate estimate of the dominance ratio even if the fission source is not converged.

\section{Hoogenboom-Martin Problem}

The final problem considered is one specified by Hoo-
Table 5 Dominance ratio results for the large 2-D problem

\begin{tabular}{ccl}
\hline Method & Mesh & Dominance ratio \\
\hline FMM & $4 \times 4$ & 0.988 \\
FMM & $9 \times 9$ & 0.993 \\
FMM & $18 \times 18$ & 0.997 \\
CMPM (MCNP) & $2 \times 2$ & $0.998(2)$ \\
PARTISN (vac. b.c.) & $360 \times 360$ & 0.99885 \\
PARTISN (extrap. b.c.) & $360 \times 360$ & 0.99846 \\
ARMA(2,1) & Not applicable & $0.9993(4)$ \\
NPMM & $4 \times 4$ & $0.9985(5)$ \\
\hline
\end{tabular}

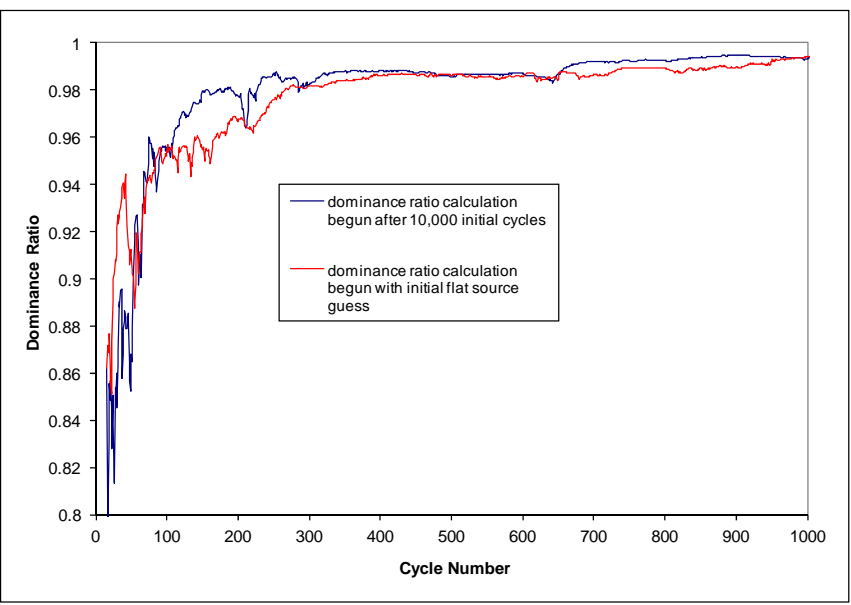

Fig. 4 Effect of discarded cycles on the dominance ratio calculation

genboom and Martin, and is an idealized representation of a large power reactor core. ${ }^{14)}$ This model consists of 241 fuel assemblies surrounded by a $20 \mathrm{~cm}$ thick steel vessel with an inner radius of $203 \mathrm{~cm}$. Each assembly consists of $17 \times 17$ pin positions, and is $400 \mathrm{~cm}$ high. There is borated water within the assemblies, between the assemblies and the reactor ves$\mathrm{sel}$, and in the regions $20 \mathrm{~cm}$ above and below the core. This model was used in a calculation that consisted of 10,000,000 neutrons per cycle, 1,000 active cycles, and 250 discard cycles. Using a $4 \times 4 \times 1$ mesh, and beginning the calculation with the first active cycle, MC21 computes a dominance ratio for this model of $0.992(6)$. To-date no other dominance ratio value for this model has been published, so there is no other result to compare to. We present this simply as an indication that the method gives a reasonable result for a very large, fairly realistic, full-core model using actual nuclear data rather than the one-group, isotropic scattering data used for the other problems. The result also provides a value for future comparisons. Figure 5 shows the dominance ratio estimate versus cycle for this problem.

\section{Conclusions}

A new expression for determining the NPM has been derived that eliminates an approximation that has figured in some of the previous literature on this topic. A new method for computing the dominance ratio in Monte Carlo reactor calculations - called the NPMM - has been described. In this method, the dominance ratio is computed by finding the 


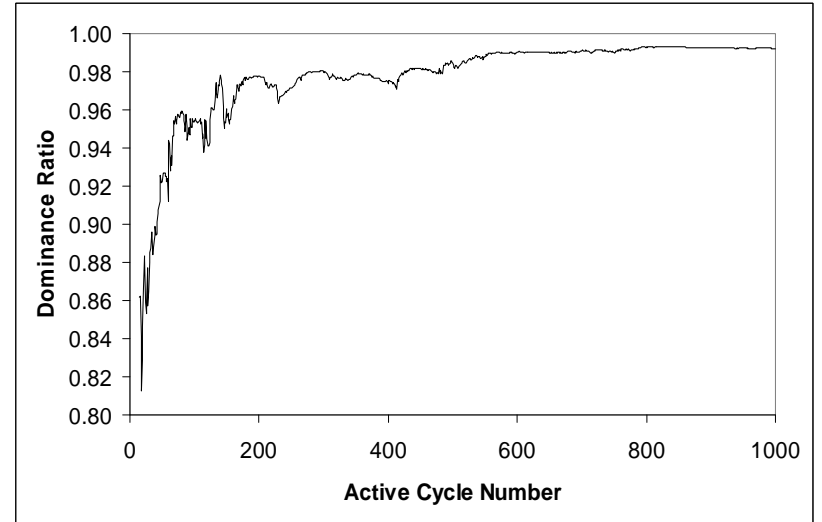

Fig. 5 Convergence of the dominance ratio for the Hoogenboom-Martin problem

largest-modulus eigenvalue of the NPM. It is shown that for consistent NPMs, the dominance ratio computed using the NPMM is identical to that computed by the CMPM. The method thus has the desirable characteristic of the CMPM that it is accurate even for a very coarse mesh. It has also been demonstrated that like the FMM, the NPMM has the potential to compute reliable estimates of the dominance ratio during the fission source convergence process.

\section{Acknowledgements}

The authors would like to acknowledge the efforts of Victoria J. Sullivan, Daniel J. Kelly and Peter S. Dobreff (all of Bechtel Marine Propulsion Corporation - Knolls Atomic Power Laboratory) in creating many of the MC21 computational models and running the associated calculations reported on in this paper. They would also like to thank D. Kent Parsons (Los Alamos National Laboratory) for a thorough review of the paper and for performing the PARTISN analyses.

\section{References}

1) T. Ueki, F. B. Brown, "Autocorrelation and dominance ratio in Monte Carlo criticality calculations," Nucl. Sci. Eng., 145, 279-290 (2003).

2) T. Ueki, F. B. Brown, D. K. Parsons, "Time series analysis of Monte Carlo fission sources-I: dominance ratio computation," Nucl. Sci. Eng., 148, 374-390 (2004).

3) B. R. Nease, T. Ueki, "Time series analysis of Monte Carlo fission sources-III: coarse-mesh projection," Nucl. Sci. Eng., 157, 51-64 (2007).

4) B. R. Nease, Time series analysis of Monte Carlo neutron transport calculations, Ph.D. dissertation, Dept. of Chemical and Nuclear Engineering, Univ. of New Mexico (2008).

5) B. Nease, F. Brown, T. Ueki, "Dominance ratio calculations with MCNP," Proc. Int. Topical Mtg. on Physics of Reactors, Interlaken, Switzerland, Sept. 14-19, 2008, Paul Scherrer Institut (2008), [CD-ROM].

6) T. Ueki, B. R. Nease, "Time series analysis of Monte Carlo fission sources-II: confidence interval estimation," Nucl. Sci. Eng., 153, 184-191 (2006).

7) E. M. Gelbard, R. Prael, "Computation of Standard Deviations in Eigenvalue Calculations," Prog. Nucl. Energy, 24, 237-241 (1990).
8) K. W. Morton, Criticality Calculations by Monte Carlo Methods, AERE T/R 1903, Atomic Energy Research Establishment (AERE) Harwell (National Archives of the UK, doc. ref. AB 15/5189) (1956).

9) T. M. Sutton, T. J. Donovan, T. H. Trumbull, P. S. Dobreff, E. Caro, D. P. Griesheimer, L. J. Tyburski, D. C. Carpenter, H. Joo, "The MC21 Monte Carlo code," Proc. Int. Conf. on Mathematics \& Computation and Supercomputing in Nuclear Applications, Monterey, California, USA, April 15-19, 2007, American Nuclear Society (2007), [CD-ROM].

10) E. Anderson, Z. Bai, C. Bischof, S. Blackford, J. Demmel, J. Dongarra, J. Du Croz, A. Greenbaum, S. Hammerling, A. McKenny, D. Sorensen, LAPACK Users' Guide ( $3^{\text {rd }} E d$.), Society for Industrial and Applied Mathematics (1999).

11) R. E. Alcouffe, R. S. Baker, J. A. Dahl, S. A. Turner, R. Ward, PARTISN: a time-dependent parallel neutral particle code system, LA-UR-05-3925 Los Alamos National Laboratory (LANL) (2005).

12) D. K. Parsons, D. E. Kornreich, "Approximations to the dominance ratio using effective and infinite multiplication results," Proc Nuclear Mathematical and Computational Sciences: A Century in Review, A Century Anew, Gatlinburg, Tennessee, USA, April 6-11, 2003, American Nuclear Society (2003), [CD-ROM].

13) G. E. P. Box, G. M. Jenkins, Time Series Analysis: Forecasting and Control, Holden-Day, Oakland, California (1976).

14) J. E. Hoogenboom, W. R. Martin, "A proposal for a benchmark to monitor the performance of detailed Monte Carlo calculation of power densities in a full size reactor core," Proc. Int. Conf. on Mathematics, Computational Methods \& Reactor Physics (M\&C 2009), May 3-7, 2009, Saratoga Springs, New York, USA, American Nuclear Society (2009), [CD-ROM].

\section{Appendix: The Connection between Various For- mulations of the Noise Propagation Matrix}

In his thesis, Nease derived two alternative formulations for the NPM. ${ }^{4)}$ The first was obtained in terms of the discretized fission source noise vector, and is given by

$$
\mathbf{A}_{0}=\mathbf{L}_{1} \mathbf{L}_{0}^{-1}
$$

where

$$
\mathbf{L}_{0} \equiv\left\langle\mathbf{e}^{(m)} \mathbf{e}^{(m) \mathrm{T}}\right\rangle
$$

and

$$
\mathbf{L}_{1} \equiv\left\langle\mathbf{e}^{(m+1)} \mathbf{e}^{(m) \mathrm{T}}\right\rangle
$$

This formulation involved no approximations beyond those inherent in Eq. (4).

His second derivation was developed to facilitate practical implementation in Monte Carlo codes, and used the fission source vector itself rather than the noise vector. This derivation introduced the additional approximation given by Eq. (12). Using the symbol $\hat{\mathbf{A}}_{0}$ to distinguish the NPM in this formulation of the NPM from that of Eq. (A1), we have

$$
\hat{\mathbf{A}}_{0}=\mathbf{L}_{1}^{\prime}\left(\mathbf{L}_{0}^{\prime}\right)^{-1}-\mathbf{I} \text {. }
$$

A third formulation has been obtained in this paper, and is given by Eq. (17). Like the formulation given by Eq. (A1), it 
involves no additional approximations beyond those used to obtain Eq. (4). Like the formulation given by Eq. (A4), however, it is given in terms of the fission source vector. For the purposes of this Appendix, this formulation is denoted $\mathbf{A}_{0}^{\prime}$, and is given by

$$
\mathbf{A}_{0}^{\prime}=\left[\mathbf{L}_{1}^{\prime}-\mathbf{S S}^{\mathrm{T}}\right]\left(\mathbf{L}_{0}^{\prime}\right)^{-1}
$$

Since the formulations given by Eqs. (A1) and (A5) were both obtained using Eq. (4) without any additional approximation, they must be equivalent. We will now show this equivalence explicitly. Using Eqs. (1), (2), (9), (10), (A2) and (A3), we may obtain the following relationships between the correlation matrices:

$$
\mathbf{L}_{0}^{\prime} \equiv N^{2} \mathbf{S}_{0} \mathbf{S}_{0}^{\mathrm{T}}+N \mathbf{L}_{0}
$$

and

$$
\mathbf{L}_{1}^{\prime} \equiv N^{2} \mathbf{S}_{0} \mathbf{S}_{0}^{\mathrm{T}}+N \mathbf{L}_{1} .
$$

Substituting Eqs. (A6) and (A7) into Eq. (A5) and applying the Sherman-Morrison formula ${ }^{\mathrm{b}}$ yields

$$
\mathbf{A}_{0}^{\prime}=\mathbf{A}_{0}\left[\mathbf{I}-\frac{N \mathbf{S}_{0} \mathbf{S}_{0}^{\mathrm{T}} \mathbf{L}_{0}^{-1}}{1+N \mathbf{S}_{0}^{\mathrm{T}} \mathbf{L}_{0}^{-1} \mathbf{S}_{0}}\right]
$$

Using $\quad \mathbf{A}_{0} \mathbf{S}_{0}=\mathbf{0}$ yields $\mathbf{A}_{0}^{\prime}=\mathbf{A}_{0}$. The NPM used in the NPMM described in this work is thus identical to the first one derived in Nease's thesis, but is computed in terms of the source vectors rather than the noise vectors. Since it was derived from an equation that is accurate to first order in the noise terms, the eigenvalue spectrum (and hence the dominance ratio) is thus also accurate to that same order.

We now turn our attention to the alternative formulation from Nease's thesis. Substituting Eqs. (A6) and (A7) into Eq. (A4) and applying the Sherman-Morrison formula yields

$$
\hat{\mathbf{A}}_{0}=\mathbf{A}_{0}+\frac{\mathbf{S}_{0} \mathbf{S}_{0}^{\mathrm{T}} \mathbf{L}_{0}^{-1}}{\mathbf{S}_{0}^{\mathrm{T}} \mathbf{L}_{0}^{-1} \mathbf{S}_{0}+\frac{1}{N}}-\mathbf{I},
$$

which in the limit of large $N$ becomes

$$
\hat{\mathbf{A}}_{0}=\mathbf{A}_{0}+\frac{\mathbf{S}_{0} \mathbf{S}_{0}^{\mathrm{T}} \mathbf{L}_{0}^{-1}}{\mathbf{S}_{0}^{\mathrm{T}} \mathbf{L}_{0}^{-1} \mathbf{S}_{0}}-\mathbf{I} .
$$

The last two terms on the right-hand side are due to the additional approximation given by Eq. (12). These terms - which are of the same order in the noise terms as is $\mathbf{A}_{0}$ itself-cause the eigenvalue spectrum of $\hat{\mathbf{A}}_{0}$ to differ significantly from that of $\mathbf{A}_{0}$. This formulation is thus unsuitable for use in the NPMM.

\footnotetext{
${ }^{\mathrm{b}}$ Gene H. Golub and Charles F. Van Loan, Matrix Computations, Johns Hopkins University Press, Baltimore (1989).
} 